\title{
Maintenance of cardiac energy metabolism by histone deacetylase 3 in mice
}

\author{
Rusty L. Montgomery, ${ }^{1}$ Matthew J. Potthoff, ${ }^{1}$ Michael Haberland,, ${ }^{1}$ Xiaoxia Qi, ${ }^{1}$ Satoshi Matsuzaki, ${ }^{2}$ \\ Kenneth M. Humphries, ${ }^{2}$ James A. Richardson,1,3 Rhonda Bassel-Duby,1 and Eric N. Olson'1
}

\begin{abstract}
1Department of Molecular Biology, University of Texas Southwestern Medical Center, Dallas, Texas, USA. 2Free Radical Biology and Aging Research Program, Oklahoma Medical Research Foundation, Oklahoma City, Oklahoma, USA. ${ }^{3}$ Department of Pathology,
\end{abstract} University of Texas Southwestern Medical Center, Dallas, Texas, USA.

\begin{abstract}
Histone deacetylase (HDAC) inhibitors show remarkable therapeutic potential for a variety of disorders, including cancer, neurological disease, and cardiac hypertrophy. However, the specific HDAC isoforms that mediate their actions are unclear, as are the physiological and pathological functions of individual HDACs in vivo. To explore the role of Hdac3 in the heart, we generated mice with a conditional Hdac 3 null allele. Although global deletion of $H$ dac 3 resulted in lethality by E9.5, mice with a cardiac-specific deletion of $H$ dac 3 survived until 3-4 months of age. At this time, they showed massive cardiac hypertrophy and upregulation of genes associated with fatty acid uptake, fatty acid oxidation, and electron transport/oxidative phosphorylation accompanied by fatty acid-induced myocardial lipid accumulation and elevated triglyceride levels. These abnormalities in cardiac metabolism can be attributed to excessive activity of the nuclear receptor PPAR $\alpha$. The phenotype associated with cardiac-specific Hdac3 gene deletion differs from that of all other Hdac gene mutations. These findings reveal a unique role for $\mathrm{Hdac} 3$ in maintenance of cardiac function and regulation of myocardial energy metabolism.
\end{abstract}

\section{Introduction}

Heart failure is a complex disorder that arises from multiple pathological insults including myocardial infarction, hypertension, and coronary artery disease. Initially, these insults are followed by an increase in cardiac mass through hypertrophy of cardiomyocytes to sustain cardiac output; however, prolonged hypertrophy often results in cardiac dilatation and failure (1-3). In addition to increases in cardiomyocyte size and organization of the sarcomere, the stressed myocardium also responds by transcriptionally reprogramming the cardiomyocyte genome to a more fetal-like gene state $(4,5)$. Multiple signaling pathways and transcription factors have been implicated in regulating hypertrophic gene activation, including GATA4, NFAT, and myocyte enhancer factor-2 (MEF2) (6).

Pharmacological inhibition of chromatin-modifying enzymes has recently emerged as an effective means of modulating pathological changes in gene expression in a variety of diseases (7). Nucleosomal histone tails can be posttranslationally modified individually or in concert with other modifications to precisely control gene expression (8). Lysine acetylation of histone tails is coupled to transcriptional activation, as the loss of positive charge relaxes chromatin, allowing for the recruitment of transcriptional machinery by DNA-bound transcription factors and subsequent gene activation (9). Acetylation of lysine residues is catalyzed by histone acetyltransferases and opposed by histone deacetylases (HDACs). HDACs remove acetyl moieties from histone tails, resulting in chromatin condensation and an overall reduction in

Nonstandard abbreviations used: ERR, estrogen-related receptor; HDAC, histone deacetylase; HDAC3cKO, HDAC3 cardiac KO; HW, heart weight; LVID, LV internal diameter; LVIDd, LVID at diastole; LVIDs, LVID at systole; MEF2, myocyte enhancer factor-2; $\alpha$-MHC, $\alpha$-myosin heavy chain; NCoR/SMRT, nuclear receptor corepressor/ silencing mediator for retinoic acid and thyroid hormone receptors; UCP2, uncoupling protein 2 .

Conflict of interest: E.N. Olson is a consultant for Gilead Sciences Inc.

Citation for this article: J. Clin. Invest. 118:3588-3597 (2008). doi:10.1172/JCI35847. transcriptional potential. The interplay between histone acetyltransferases and HDACs allows for rapid changes in gene expression in response to extrinsic or intrinsic signals and, therefore, has become a therapeutic target for a variety of pathological states, including cancer and cardiac disease.

Studies in mice and cultured cardiomyocytes have identified both class I and class II HDACs as key regulators of cardiac growth and disease (10). Class II HDACs bind and repress MEF2 under normal physiologic conditions; however, in response to hypertrophic stimuli, these HDACs become phosphorylated and are exported from the nucleus, allowing for the derepression of MEF2 and other transcription factors $(11,12)$. Paradoxically, inhibition of HDACs is able to blunt the hypertrophic response, which suggests a role for class I HDACs in cardiac hypertrophy (13-15).

The 4 class I HDACs (HDAC1, HDAC2, HDAC3, and HDAC8) share extensive homology and are widely expressed (16), but little is known of their individual functions in vivo. Previously, we showed that cardiac deletion of either $H d a c 1$ or Hdac2 in the heart did not affect cardiac structure or function, whereas deletion of both of these Hdacs resulted in perinatal lethality from cardiac arrhythmias accompanied by dilated cardiomyopathy and upregulation of genes encoding skeletal muscle-specific contractile proteins and calcium channels (17). Using a lacZ enhancer trap allele of Hdac2, other investigators showed that $H d a c 2$ mutant mice are viable but resistant to pathological hypertrophy (18).

To examine the function of other class I HDACs during cardiac growth and disease, we generated mice with a conditional null allele of Hdac3. While global deletion of Hdac 3 resulted in embryonic lethality prior to E9.5, cardiac-specific deletion of Hdac3 resulted in cardiac hypertrophy and fibrosis by 3 months of age and lethality by 4 months. These mice showed upregulation of genes involved in fatty acid uptake and oxidation, downregulation of the glucose utilization pathway, and ligand-induced myocardial lipid accumulation due to increased activity of the nuclear 
A
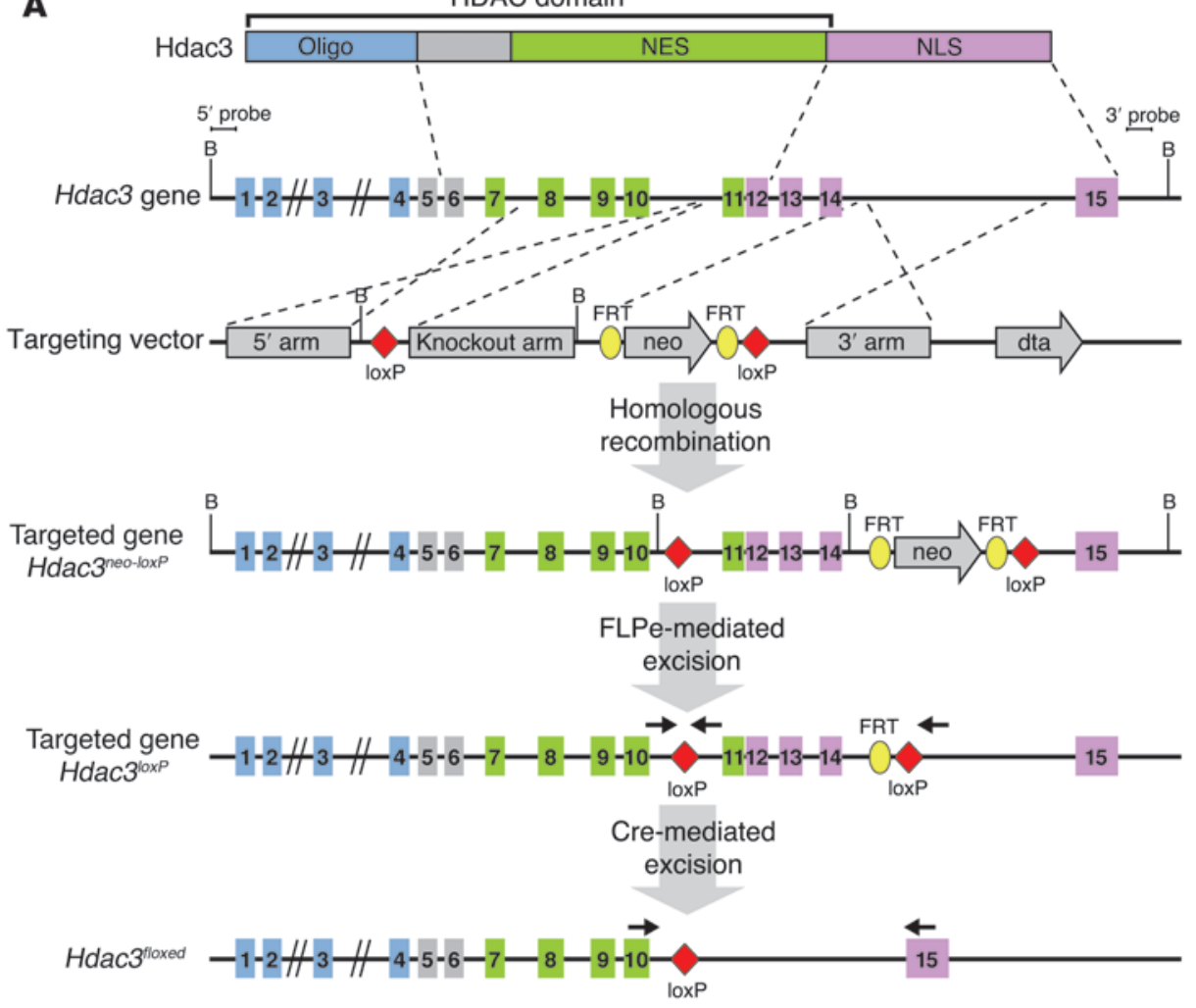

B

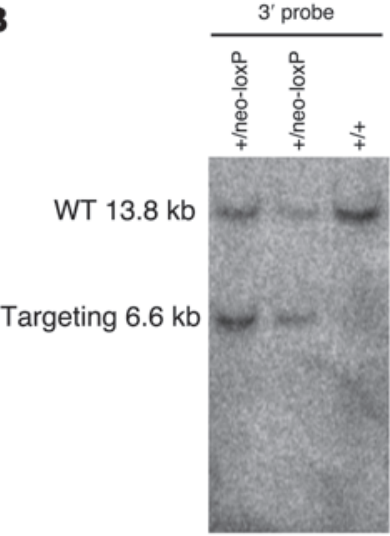

HDAC domain

Figure 1

Generation of a conditional Hdac3 allele. (A) Strategy to generate a conditional Hdac3 allele. Protein, genomic structure, targeting vector, and targeted allele are shown. IoxP sites were inserted upstream of exon 11 and downstream of exon 14. The neomycin cassette was removed by crossing to FLPe transgenic mice. Cre-mediated excision leaves 1 loxP site in place of exons 11 through 14. NES, nuclear export sequence; NLS, nuclear localization sequence; FRT, flipase recognition target; dta, diphtheria toxin A. (B) Representative Southern blot of genomic DNA to show germ-line transmission. WT $(\sim 13.8 \mathrm{~kb})$ and targeted $(\sim 6.6 \mathrm{~kb})$ bands are indicated. (C) Genotyping of Hdac3 conditional mice by genomic PCR. Primer triplex includes 1 set flanking the $5^{\prime}$ loxP site and a third primer downstream of the $3^{\prime}$ loxP site. Global deletion by CAG-Cre removes the primer within the loxP sites, resulting in 1 fragment of approximately $650 \mathrm{bp}$. Primers are shown in $\mathbf{A}$. hormone receptor PPAR $\alpha$, a well-known regulator of metabolism. Additionally, these hearts showed mitochondrial dysfunction and decreased cardiac efficiency. Our findings reveal distinct functions for different class I HDAC isoforms in the heart and suggest $\mathrm{Hdac} 3$ functions as an independent regulator of cardiac growth and myocardial energy metabolism.

\section{Results}

Conditional deletion of Hdac3. Knowing of the embryonic and neonatal lethality associated with loss of Hdac1 or Hdac2, we generated a conditional null allele of $H d a c 3$ to investigate the role of $\mathrm{Hdac} 3$ in the adult heart. Targeting of $\mathrm{Hdac} 3$ was performed by introducing loxP sites upstream of exon 11 and downstream of exon 14 through homologous recombination (Figure 1A). This mutation deletes almost all of the nuclear import sequence and a carboxyterminal region that is necessary for transcriptional repres- sion (19). Germ-line transmission was detected by Southern blot, and deletion of Hdac3 was confirmed at the genomic level (Figure 1C). Hdac $3^{\text {neo-loxP }}$ mice were bred to CAG-Cre (20) transgenic mice, which express Cre recombinase ubiquitously, allowing for the generation of $\mathrm{Hdac}^{+/-}$mice. $\mathrm{Hdac} 3^{+/-}$mice were intercrossed to obtain $\mathrm{Hdac}^{-/-}$mice, which died before E9.5 due to defects in gastrulation (data not shown).

Cardiac deletion of Hdac 3 causes cardiac bypertrophy. To circumvent embryonic lethality, we deleted Hdac3 specifically in the heart by breeding homozygous $H$ dac $3^{\text {loxP/loxp }}$ mice to transgenic mice expressing Cre recombinase under the control of the $\alpha$-myosin heavy chain $(\alpha-M H C)$ promoter (21). Cardiomyocyte-restricted deletion of Hdac3 (hereafter referred to as Hdac3cko for Hdac3 cardiac KO; wild-type are represented by $H d a c 3^{l o x P} /$ loxP mice) was confirmed by RT-PCR and Western blot (Figure 2, A and B). RT-PCR of Hdac3 using primers flanking the floxed region showed efficient 
A

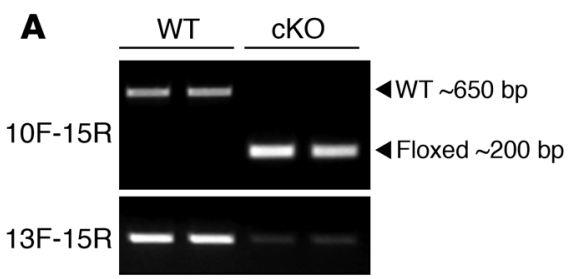

B

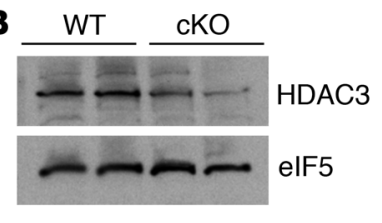

\section{Figure 2}

Cardiac-specific deletion of Hdac3. (A) Semiquantitative RT-PCR showing Hdac3 transcript levels in wild-type and Hdac3cko mice using primers in exon 10, forward $(F)$, and exon 15, reverse $(R)$, or exon 13, forward, and exon 15, reverse. Cardiac deletion of $\mathrm{Hdac} 3$ results in deletion of exons 11 through 14. (B) Western blot showing reduced expression of Hdac3 in Hdac3cko hearts. (C) Real-time PCR of class I and class II Hdacs in wild-type and Hdac3cko hearts. cKO, Hdac3cko.

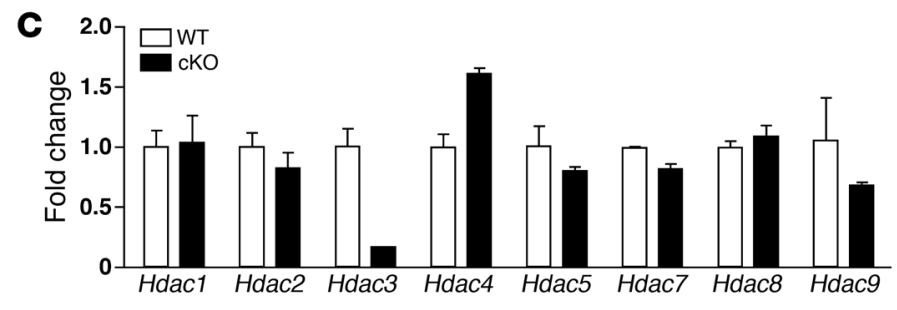

deletion of $H d a c 3$; however, residual expression of $H d a c 3$ was seen using primers within the deleted region as well as by Western blot, suggesting that $\mathrm{Hdac} 3$ is expressed at very low levels in $\alpha-M H C-C r e$ negative cell types such as cardiac fibroblasts (Figure 2, A and B). Expression levels of other class I and class II HDACs were not significantly altered in Hdac3cko hearts (Figure 2C).

Hdac3cko mice were born at Mendelian ratios; however, signs of cardiac hypertrophy, assessed by heart weight (HW) to BW ratios, were apparent by 4 weeks of age and were exacerbated by 12 weeks of age, resulting in a $72 \%$ increase in $\mathrm{HW} / \mathrm{BW}$ ratio compared with wild-type littermates (Figure 3A). Cardiac deletion of Hdac3 resulted in $100 \%$ lethality by 16 weeks of age, with significant lethality occurring between 12 and 14 weeks (Figure 3B). Hearts of Hdac3cko mice were hypertrophic and showed enlargement of both right and left atria (Figure 3C). Histology confirmed cardiomyocyte hypertrophy, especially in the LV free wall and septum, as well as robust interstitial fibrosis in Hdac3cko mice compared with wild-type littermates (Figure 3C). Cardiac stress markers atrial natriuretic factor $(A N F, N p p a)$, brain natriuretic peptide $(B N P, N p p b)$, and $\alpha$-skeletal actin (Acta1) were significantly upregulated as early as 8 weeks of age in mutant mice, consistent with the hypertrophy seen by histology (Figure 3D). Expression of p21 (Cdkn1a), shown to be repressed by class I HDACs in a variety of cell types (22), was also significantly upregulated in hearts of Hdac3cko mice, supporting the role of class I HDACs as transcriptional repressors of 221.

Ultrastructural analysis of the LV free wall of the adult myocardium revealed that the normal juxtaposition of sarcomeres to mitochondria (Figure 3E), which facilitates efficient myofibrillar contraction and relaxation in normal cardiomyocytes, was aberrant in Hdac3cko mutants. Instead, cardiac deletion of Hdac3 resulted in disorganized and fragmented myofibrils, associated with intracellular debris and disarrangement of mitochondria that showed reduced cristae density (Figure 3E).

To determine whether there is a correlation between Hdac3 expression levels and pathological conditions of the heart, we examined Hdac3 expression in multiple settings of hypertrophy and failure. Hdac3 levels were not significantly altered following angiotensin infusion, aortic banding, myocardial infarction, or in the Zucker diabetic fatty (ZDF) rat heart; however, Hdac3 expression was decreased following isoproterenol infusion (Supplemental Figure 1; supplemental material available online with this article; doi:10.1172/JCI35847DS1).
Functional analyses of wild-type and Hdac3cko mice were performed at 12 weeks of age by echocardiography. As shown in Figure 4, Hdac3cko mice showed diminished contractility and ventricular dysfunction, as indicated by reduced fractional shortening ( $39.25 \pm 0.75$ vs. $78.65 \pm 4.35$ for WT), and increased LV chamber dilatation, as assessed by systolic and diastolic internal diameters, LVIDs and LVIDd, respectively. Additionally, ECG was performed on 8-and 14-week-old mice to determine whether Hdac3cko mice have conduction system defects. Hdac3cko mice showed no overt abnormalities in their sinus rhythm compared with wild-type littermates (Supplemental Figure 2). Furthermore, continuous telemetry was performed on wild-type and Hdac3cko mice from 12 to 16 weeks of age to determine whether arrhythmias contributed to sudden death. No signs of cardiac arrhythmia were observed in Hdac3cko mice when compared with wild-type littermates (data not shown).

Upregulation of myocardial energetic genes from cardiac deletion of Hdac3. In an effort to more precisely understand the primary cause of cardiomyopathy in Hdac3 mutant hearts, we performed microarray analysis on LVs from 5-week-old mice. At this time, mutant hearts showed moderate increases in HW/BW ratios and relatively minor changes in cardiac stress markers (Figure 3A and data not shown). Gene ontology analysis of significantly upregulated transcripts in Hdac3cko mice revealed dramatic dysregulation of cardiac metabolism in the mutant hearts (Figure 5A and Supplemental Data).

Cardiac energetics is tightly regulated by the PPAR and the estrogen-related receptor (ERR) families of nuclear hormone receptors (23), and PPAR $\alpha$ cardiac overexpression results in diabetic cardiomyopathy (24). Expression levels for PPAR $\alpha, \operatorname{PPAR} \gamma$, ERR $\alpha$, and PGC- $1 \alpha$ were unchanged in Hdac3cko hearts compared with wild-type littermates, suggesting the phenotype is independent of changes in receptor or coactivator expression (Supplemental Figure 3). To determine whether the cardiac hypertrophy and ventricular dysfunction in Hdac3 mutant mice resulted from rampant nuclear receptor-dependent gene activation, we assayed known PPAR $\alpha$ target genes in ventricles of Hdac3cko mice. PPAR $\alpha$ has been shown to regulate expression of the mitochondrial uncoupling proteins UCP2 and UCP3 (25). Accordingly, transcript levels for both UCP2 and UCP3 were significantly upregulated at baseline (6.5-fold and 2.9-fold, respectively), and this induction was increased upon administration of Wy14,643, a synthetic PPAR $\alpha$-agonist (Figure 5B). 

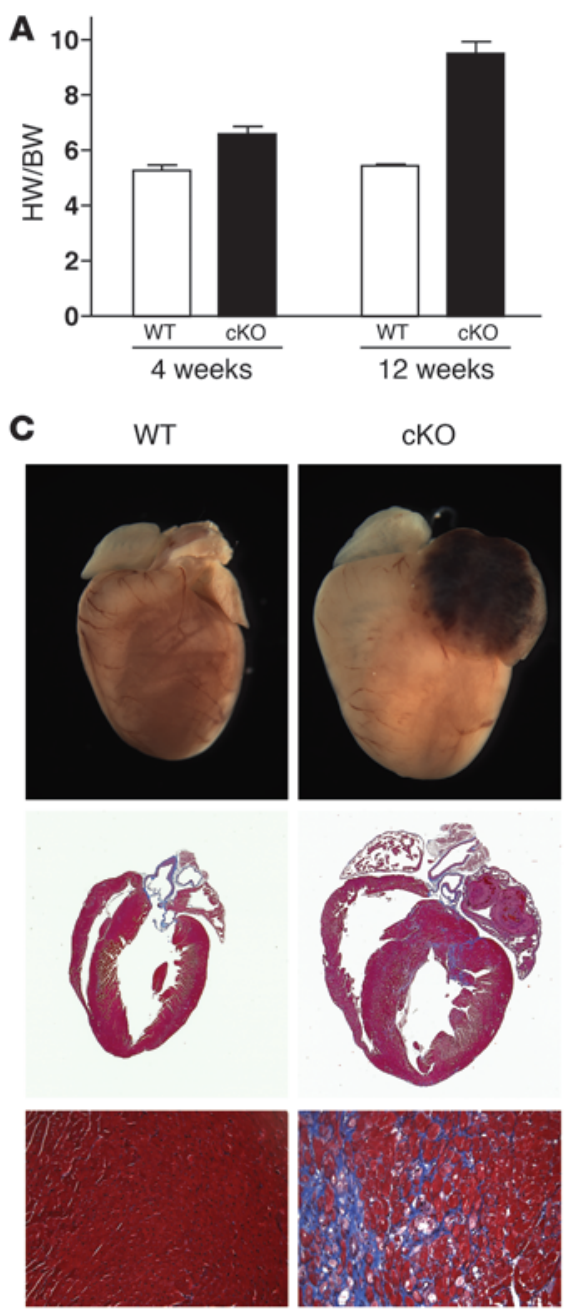

B

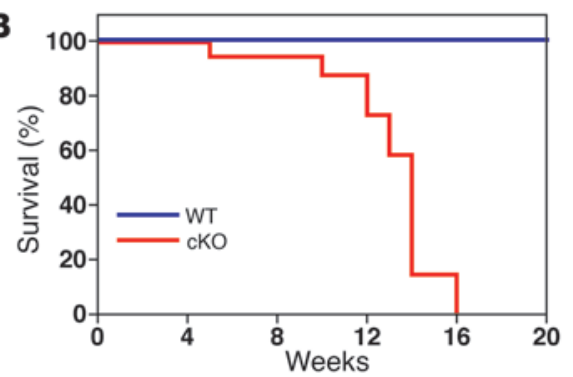

D

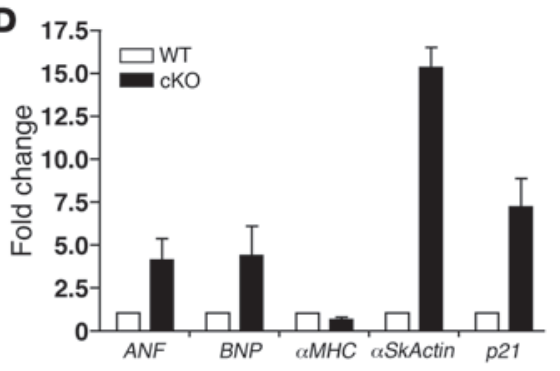

E
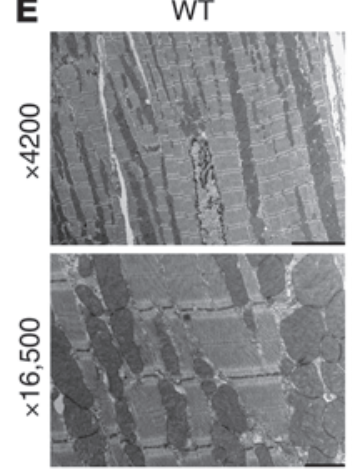

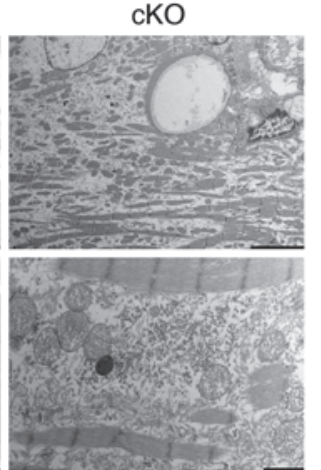

\section{Figure 3}

Cardiac defects resulting from cardiac deletion of $\mathrm{Hdac} 3$. (A) HW/BW ratios of WT and Hdac3cko showing progression of cardiac hypertrophy. (B) Kaplan-Meier survival curve showing lethality by 16 weeks in Hdac3cko mice. (C) Masson trichrome-stained sections of wild-type and Hdac3cko mice at 12 weeks. Deletion of $\mathrm{Hdac} 3$ results in cardiac hypertrophy, left atrial thrombus, and cardiac fibrosis, seen in blue. Original magnification, $\times 20$ (lower panels). (D) Expression of cardiac stress markers in Hdac3cko mice at 8 weeks. mRNA transcript levels were detected by real-time RT-PCR and normalized to $18 \mathrm{~S}$ ribosomal RNA. ANF, atrial natriuretic factor; BNF, brain natriuretic peptide; $\alpha$ SkActin, $\alpha$-skeletal actin. (E) Electron microscopy of LV tissue from WT and Hdac3cko hearts at 8 weeks. Scale bars: $5000 \mathrm{~nm}(\times 4,200) ; 1000$ $\mathrm{nm}(\times 16,500)$.
Real-time PCR analysis of genes encoding proteins involved in fatty acid import, transport, and esterification (fatty acid transport protein [FATP, Slc27a1], CD36, and fatty acyl-CoA synthetase [FACS, Acsl1]) showed modest to insignificant changes at baseline. Following administration of Wy14,643, these levels were upregulated (Figure $5 \mathrm{C}$ ), consistent with previous studies showing PPAR ligands to be rate limiting under physiological conditions (24).

The expression of PPAR $\alpha$-responsive genes involved in fatty acid oxidation was also analyzed by real-time PCR. Similar to the expression of PPAR $\alpha$-dependent genes involved in fatty acid import, induction of PPAR $\alpha$-responsive genes involved in fatty acid oxidation was modest at baseline. Surprisingly, expression levels of muscle carnitine palmitoyl transferase-1 (M-CPT1) were unchanged in Hdac3cko mice compared with wild-type mice, and Wy14,643 treatment had no effect on $M$-CPT1 transcript levels in wild-type or Hdac3cko hearts (Figure 5D); however, additional enzymes involved in mitochondrial fatty acid oxidation were significantly upregulated at baseline, including long- and very long-chain acyl-CoA dehydrogenase ( $L C A D, A c a d l$, and $V L C A D, A c a d v l$, respectively) (data not shown). Conversely, acyl-CoA oxidase 1 ( $A C O X)$ was significantly increased in $H$ dac3cko hearts and was further upregulated in response to Wy14,643 (Figure 5D). ACOX is the first enzyme in peroxisomal fatty acid $\beta$-oxidation, suggesting Hdac $3 c k o$ hearts possess greater fatty acid oxidation potential than wild-type littermates.
Decreased expression of genes involved in glucose utilization from cardiac deletion of Hdac3. In diabetic cardiomyopathies, increased expression of genes involved in fatty acid import and fatty acid oxidation is coupled to a decreased utilization of the glucose oxidation pathway (26). To determine whether Hdac3cko mice show defects in glucose uptake and utilization, we examined expression levels of the glucose transporters GLUT1 and GLUT4. GLUT1 levels were relatively unchanged in $H d a c 3 c k o$ mice compared with wild-type (data not shown), whereas GLUT4 expression was significantly downregulated in Hdac3cko mice (Figure 5E) and was further decreased in response to Wy14,643 (Figure 5E). GLUT1 controls basal glucose uptake while GLUT4 regulates glucose transport in an insulin-sensitive-dependent manner. Downregulation of GLUT4 in Hdac3cko mice is consistent with the phenotype resulting from excessive PPAR $\alpha$ activity.

Pyruvate dehydrogenase kinase 4 (PDK4) regulates the pyruvate dehydrogenase complex through phosphorylation and subsequent inactivation. PDK4 levels and activity are increased in diabetic hearts (27). Similarly, Hdac3cko mice showed an increase in expression of $P D K 4$ that was further elevated upon administration of Wy14,643 (Figure 5E).

Localization of Hdac3 to the promoters of PPAR $\alpha$-responsive genes. To further investigate the regulation of PPAR $\alpha$-responsive genes by $\mathrm{Hdac} 3$, we performed ChIP on multiple genes upregulated in 

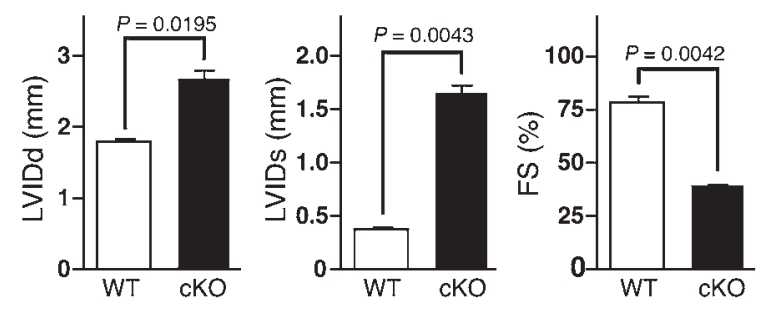

Hdac3cko hearts from neonatal rat myocytes. Immunoprecipitation of Hdac3 or PPAR $\alpha$ was able to robustly pull down the PPAR response element within the promoters of the UCP2, UCP3, FACS, $F A T P$, and $P D K 4$ genes compared with the negative anti-HA control or the negative exon 5 of UCP2 control (Figure 6A). These findings indicate that $\mathrm{Hdac} 3$ resides in a repressive complex with PPAR $\alpha$ under basal physiological conditions.

Histone acetylation is locally increased in Hdac3cko hearts. To determine whether loss of Hdac3 results in global changes in histone acetylation, we isolated histones from wild-type and Hdac3cko hearts and examined the acetylation states of histones H3 and H4. Western blot analyses for acetyl-H3, acetyl-H4, and pan-acetyllysine revealed no global changes in histone acetylation in Hdac3cko hearts (Figure 6B). The localization of Hdac3 to the promoters of multiple PPAR-responsive genes suggested that changes in histone acetylation might occur locally at the promoters of upregulated transcripts. To investigate this, we isolated cardiomyocytes from wild-type and Hdac3cko mice and performed quantitative ChIP assays. Immunoprecipitation on the PPAR-responsive elements with acetyl$\mathrm{H} 3$ revealed increased local acetylation levels at the promoters of multiple PPAR-responsive genes in Hdac3cko myocytes compared with wild-type controls but not on the promoters of genes unaltered in Hdac3cko, such as Gapdh (Figure 6C).

\section{Figure 5}

Aberrant expression of cardiac metabolism genes from cardiac deletion of Hdac3. (A) Microarray analysis was performed at 5 weeks, and gene ontology analysis was performed with PANTHER (http://www.pantherdb.org). Significantly enriched biological processes are shown and plotted as the -log ( $P$ value). (B) Expression of PPAR-regulated mitochondrial uncoupling genes is increased in Hdac3cko mice. (C and D) Expression of fatty acid uptake and oxidation genes is moderately increased in Hdac3cko hearts. FATP, fatty acid transport protein. (E) Glucose metabolism is decreased in Hdac3cko hearts. For B-E, real-time RT-PCR was performed from LV RNA of 6-week-old wild-type and Hdac3cko mice in absence or presence of Wy14,643. Transcript levels were normalized to $18 \mathrm{~S}$ ribosomal RNA. Error bars represent SD.

A

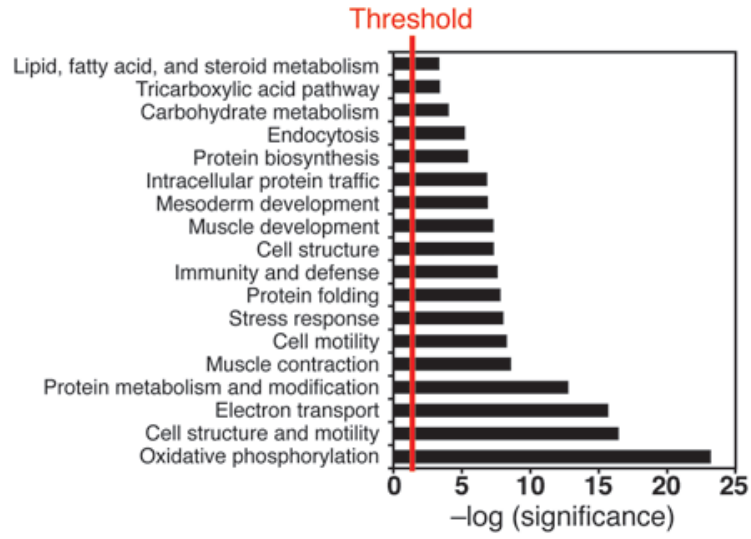

B

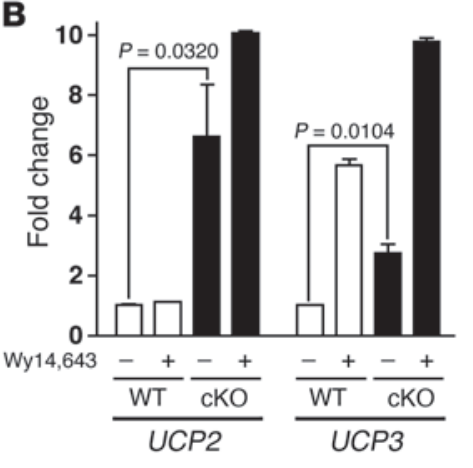

D

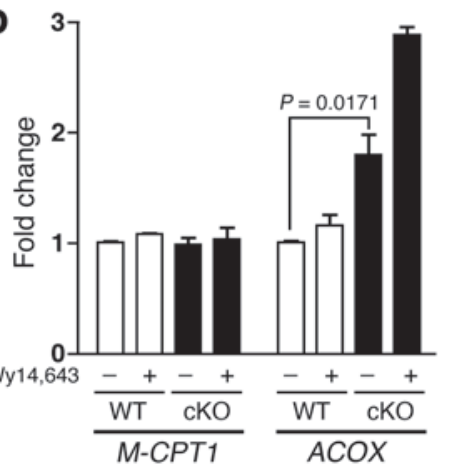

\section{Figure 4}

Echocardiographic data of Hdac3cko mice. Values show severe ventricular dysfunction in Hdac3cko hearts at 12 weeks of age. Values represent mean \pm SEM. FS, fractional shortening.
Cardiac deletion of Hdac3 causes ligand-induced lipid accumulation. The ligand-inducible activation of multiple PPAR-responsive genes in cardiomyocytes of Hdac3cko mice suggests these mice are sensitive to increased fatty acids in the circulation. A hallmark of the diabetic heart is lipid accumulation in myocytes due to augmented fatty acid import (28). To determine whether Hdac3 mutant mice show myocardial lipid accumulation following increases in circulating fatty acids, we subjected $H d a c 3 c k o$ and wild-type mice to a 24-hour

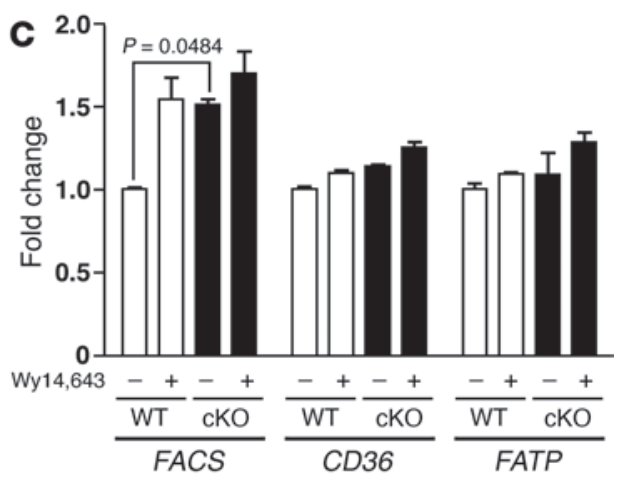

$\mathbf{E}$

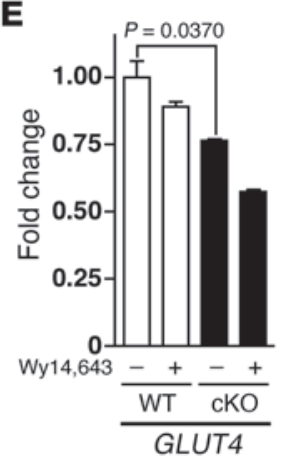

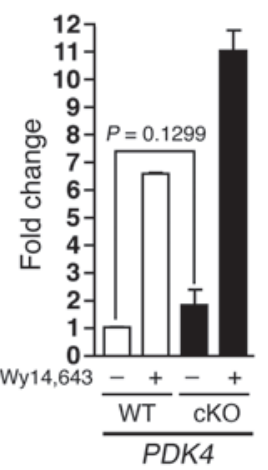



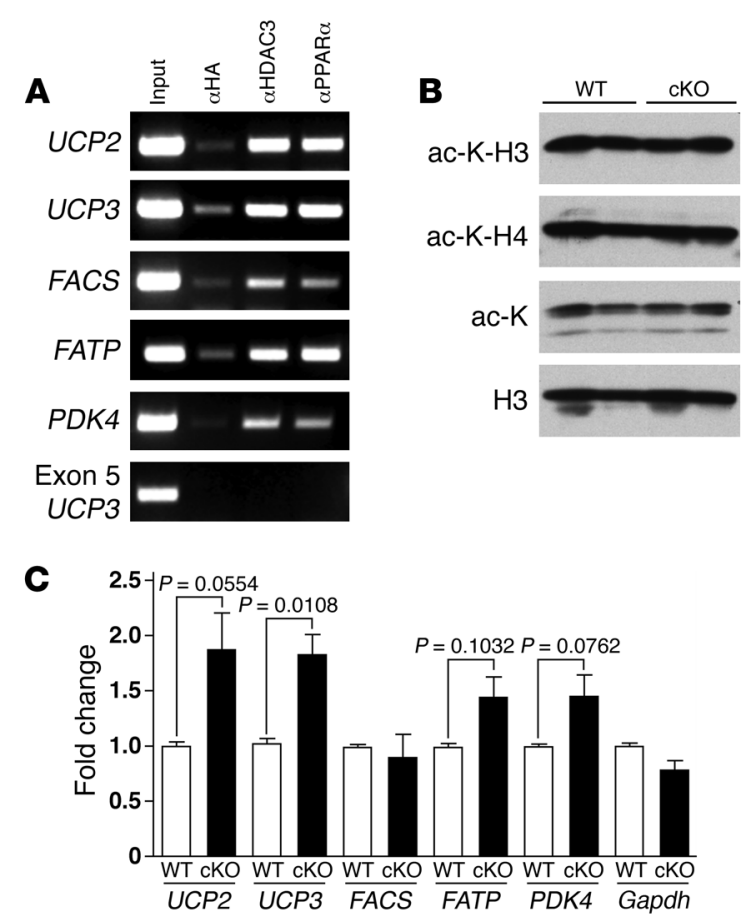

Figure 6

Local promoter architecture of dysregulated transcripts. (A) ChIP assays were performed from neonatal rat myocytes. Chromatin was immunoprecipitated with antibodies against HA, HDAC3, or PPAR $\alpha$. Primers flank the PPAR-responsive elements of each gene, and precipitated DNA was analyzed by PCR. Nonimmunoprecipitated sample served as an input control. (B) Global histone acetylation is unchanged in Hdac3cko hearts. Histones were isolated from wild-type and Hdac3cko hearts and subjected to Western blot analysis using antibodies against acetyl-H3 (ac-K-H3), acetyl-H4 (ac-K-H4), pan-acetyl-lysine (ac-K), and H3. (C) Representative quantitative ChIP on dysregulated transcripts performed in triplicate from myocytes isolated from wild-type and Hdac3cko hearts using anti-acetyl-H3 for immunoprecipitation.

fast, which induces circulating fatty acids that can subsequently serve as ligands for PPARs. After fasting, hearts were excised and triglycerides were quantified. Mice fed ad libitum showed no significant difference in triglyceride content between Hdac3cko and wild-type mice (Figure 7A). However, Hdac3cko fasted mice showed a dramatic increase in myocardial triglycerides compared with wild-type controls (Figure 7A). Quantification of serum fatty acid and triglyceride levels showed there to be no significant difference between wild-type and Hdac3cko mice (data not shown). Consistent with these findings, oil red $\mathrm{O}$ staining of histological sections from fed and fasted wild-type and Hdac3cko hearts showed no significant staining in fed mice of either genotype (data not shown), whereas fasted myocytes from Hdac3cko mice showed a pronounced increase in neutral lipid accumulation (Figure 7B). Neutral lipids could be readily visualized throughout both ventricular free walls as well as the ventricular septum; however, the atria remained free of lipids. These results further point to Hdac3 as an important regulator of PPAR and other transcription factors that govern myocardial energy metabolism.

Mitochondrial dysfunction resulting from cardiac deletion of Hdac3. To further define the metabolic abnormalities resulting from cardiacspecific deletion of $H d a c 3$, we compared mitochondrial function in wild-type and Hdac3cko mice. Mitochondrial dysfunction is intimately linked to diabetic cardiomyopathy through defects in glucose utilization and increased fatty acid oxidation (29). Increased fatty acid oxidation results in increased reducing equivalents to the electron transport chain, which in turn generates free radicals and leads to mitochondrial uncoupling (30). Free fatty acids are able to directly promote free radical production through the inhibition of complex I of the electron transport chain (31-33). Consistently, Hdac3cko mice showed a $25 \%$ reduction in complex I activity accompanied by a $39 \%$ reduction in NADH oxidase activity (Figure 7C). Additionally, free radical production was increased 2 -fold in Hdac3cko mice compared with wild-type littermates (Figure 7C).

\section{Discussion}

The results of this study reveal a unique and unexpected role of Hdac 3 as a key regulator of cardiac energy metabolism. The severe hypertrophy and metabolic abnormalities associated with deletion of $H d a c 3$ contrast with the lack of a cardiac phenotype upon deletion of either Hdac1 or Hdac2 or the ventricular dilatation without metabolic abnormalities seen in mice with combined deletion of both Hdac1 and Hdac2 (17). Thus, despite the high degree of homology among Hdac1, Hdac2, and Hdac3, Hdac3 clearly plays a unique role in maintenance of cardiac function.

Hdac3 as a regulator of cardiac energy metabolism. Maintenance of myocardial energy metabolism requires a precise balance of nuclear receptor activation and repression. The PPAR and ERR families of nuclear hormone receptors control cardiac energetics through the activation of target genes to meet the metabolic demands of the heart during stress (23). Under basal conditions, Hdac3, together with the nuclear receptor corepressor/silencing mediator for retinoic acid and thyroid hormone receptors (NCoR/SMRT) or the retinoblastoma $(\mathrm{Rb})$ complex, is specifically recruited by PPARs and other nuclear receptors to the promoters of target genes to facilitate transcriptional repression by nuclear receptors $(34,35)$. PPAR $\alpha$ and PPAR $\delta$ function as central regulators of cardiac fatty acid metabolism $(24,36-38)$. Consistent with our phenotype, cardiac-specific overexpression of PPAR $\alpha$ causes increased expression of genes associated with mitochondrial uncoupling, fatty acid uptake, and oxidation and concomitant decreased expression of genes associated with glucose uptake, mimicking diabetic cardiomyopathy (24). Surprisingly, cardiac-specific overexpression of PPAR $\delta$ results in a metabolic phenotype independent of that of overexpression of PPAR $\alpha$, indicating that separate PPAR isoforms control specific metabolic programs in the adult heart (39). Furthermore, PPAR $\delta$ overexpression does not result in cardiomyopathy or ligand-induced lipid accumulation, implying that the phenotype from loss of Hdac3 in cardiomyocytes is likely due to rampant PPAR $\alpha$ activation and not other PPAR family members. Nevertheless, we cannot rule out the additional activation of other nuclear receptors (such as ERR $\alpha$ ) that depend upon Hdac3 and NCoR/SMRT to mediate repression. While ERR $\alpha$ and PPAR $\alpha$ coregulate multiple downstream target genes, ERR $\alpha$ has been shown to directly induce PPAR $\alpha$ expression and PPAR $\alpha$ is required for ERR $\alpha$ induction of $\beta$-oxidation genes (40). PPAR $\alpha$ expression is unchanged in Hdac3cko hearts, suggesting that metabolic derangements in Hdac3cko hearts primarily arise from PPAR $\alpha$ activity and not ERR $\alpha$; however, we are currently investigating other ERR $\alpha$ dependent mechanisms in Hdac3cko mice. Additionally, Hdac3 has recently been shown to regulate cholesterol synthesis independent of nuclear receptors, such as through the recruitment of YY1 (41). 
A

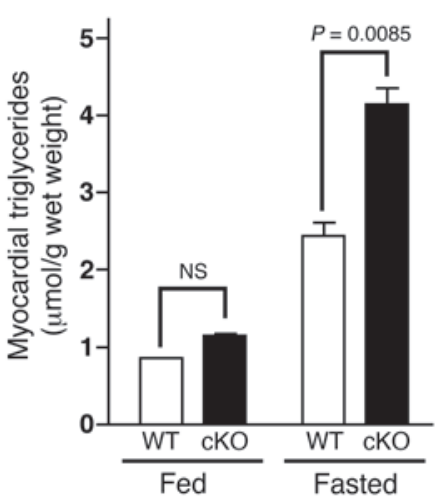

B

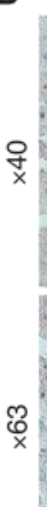

WT

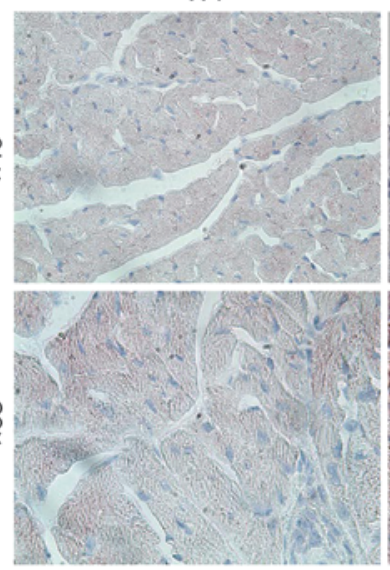

cKO

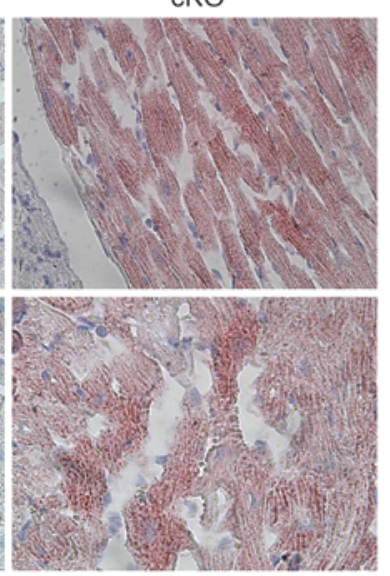

\section{Figure 7}

Myocardial lipid accumulation and mitochondrial dysfunction in Hdac3cko mice. (A) Increased triglycerides in Hdac3cko hearts after fasting. Triglycerides were extracted from wildtype and $\mathrm{Hdac} 3 c k$ hearts at 8 weeks and quantified. (B) Oil red $O$ staining of 8-week-old wild-type mice and cardiac deletion of $\mathrm{Hdac} 3$ following a 24-hour fast. Red droplets indicate neutral lipids. Hdac3cko mice show substantial lipid accumulation. (C) Hdac3cko hearts show impaired mitochondrial function. Complex I activity, NADH oxidase activity, and free radicals were determined from 8-week-old wild-type and Hdac3cko mice.
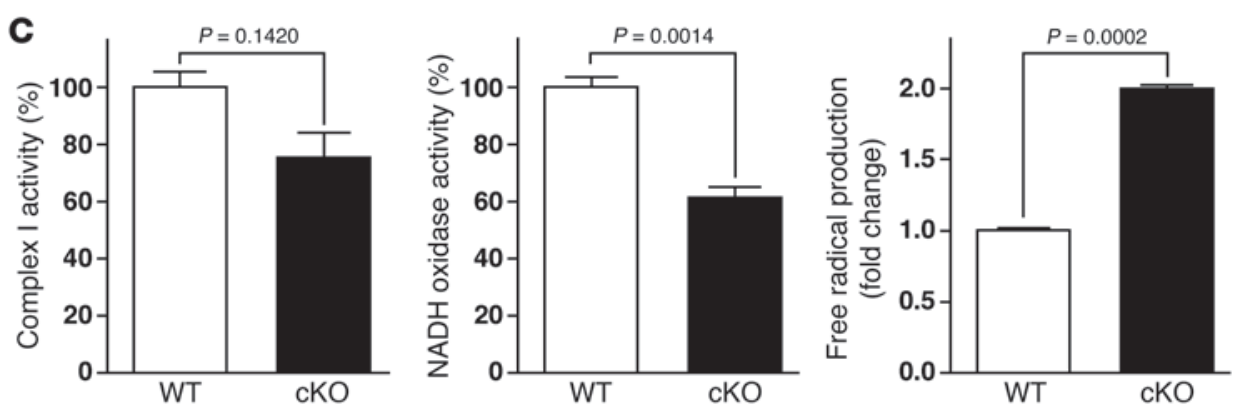

Current experiments are underway to identify additional transcription factors contributing to the metabolic derangements from loss of Hdac 3 in cardiomyocytes.

The mitochondrial derangements from loss of Hdac 3 in cardiomyocytes also resemble those seen in diabetic cardiomyopathies. The increased oxidation of fatty acids in diabetic hearts results in augmented reducing equivalents to the electron transport chain, resulting in free radical production, mitochondrial uncoupling, and decreased cardiac efficiency (30). The increase in UCP2 and $U C P 3$ expression suggests that mitochondria of Hdac3cko mice are uncoupled, and the increased free radical production and defects in electron transport are potential contributors to the ventricular dysfunction. Given the increase in expression of genes involved in oxidative phosphorylation and electron transport, as revealed by microarray analysis, these findings suggest either that $\mathrm{Hdac} 3$ directly regulates these genes through ERR $\alpha$ or additional mechanisms or that a feedback mechanism exists that activates these genes to compensate for the electron transport chain inhibition and reduction in cardiac efficiency. Generation of reactive lipid intermediates from mitochondrial dysfunction in diabetic cardiomyopathies has been proposed as a "lipotoxic" mechanism of lipidinduced myocyte death leading to cardiac dysfunction. Surprisingly, no difference in apoptosis was observed between Hdac3cko and wild-type mice by TUNEL analysis (data not shown); however, this does not rule out additional mechanisms of cell loss that are currently being investigated.

Distinct roles of class I HDACs in cardiac growth and disease. The abnormalities in cardiac metabolism resulting from loss of Hdac3 in cardiomyocytes contrasts with the phenotypes resulting from genetic deletion of other class I HDACs. Deletion of Hdac1 or Hdac2 indi- vidually in cardiomyocytes causes no phenotypic abnormalities; however, deletion of Hdac1 and Hdac2 together in cardiomyocytes results in lethality by 2 weeks of age, accompanied by arrhythmias, dilated cardiomyopathy, and misexpression of calcium channels and contractile proteins (17). Mice lacking Hdac1 and Hdac2 also show no metabolic abnormalities, further illustrating the distinct functions of Hdac3 versus the functionally redundant Hdac1 and Hdac2 enzymes in cardiac growth and development. Hdac3 has classically been considered distinct from Hdac1 and Hdac2 in transcriptionally repressive complexes, associating with $\mathrm{NCoR} /$ SMRT as opposed to the corepressor to RE1 silencing transcription factor (CoREST), Sin3, and nucleosomal remodeling and deacetylation (NuRD) complexes that contain Hdac1 and Hdac2 (16). The results of this study further support differing repressive complexes in vivo for class I HDACs.

Other studies have indirectly implicated Hdac3 in the control of cardiac growth. Class II HDACs act as antihypertrophic mediators through the binding and repression of MEF2 and other transcription factors in the adult heart (10-11); however, class II HDACs do not possess intrinsic deacetylase activity but instead recruit the NCoR/SMRT-Hdac3 complex to mediate deacetylation and transcriptional repression (42). Additionally, Hdac3 has been shown to directly bind and deacetylate MEF2, thereby regulating MEF2 activity (43). Cardiac-specific overexpression of MEF2 results in cardiomyopathy with extensive fibrosis (44); however, MEF2 activity was only slightly increased in hearts of Hdac3cko mice as assayed by crossing Hdac3cko mice to reporter mice harboring a MEF2-dependent transgene (Supplemental Figure 4). These results indicate that the metabolic derangements and cardiomyopathy associated with loss of Hdac3 are unlikely to be due to 
excessive MEF2 activity but rather to rampant nuclear receptordependent activation. It should also be pointed out that Hdac3cko mice do not phenocopy all aspects of diabetic cardiomyopathy. The excessive fibrosis seen in Hdac3cko hearts, for example, contrasts with previous mouse models of diabetic cardiomyopathy. Multiple collagens and extracellular matrix proteins are significantly upregulated in the Hdac3cko hearts (Supplemental Data), however it is currently unclear whether these are direct targets of $\mathrm{Hdac} 3$ or, alternatively, whether they are upregulated as a secondary consequence of cardiac dysfunction.

The distinct phenotypes associated with loss-of-function studies of class I HDACs in the heart underscore the necessity for more thorough analyses of specific roles of individual class I HDACs in cardiac physiology and pathology. Deletion of Hdac1 or Hdac2 individually in cardiomyocytes did not affect the response to hypertrophic stimuli compared with wild-type littermates; however, mice from a gene-trap deletion of Hdac2 fail to undergo cardiac hypertrophy during aortic constriction or $\beta$-adrenergic stimulation $(17,18)$, potentially pointing to a role for Hdacs in cardiac fibroblasts as a mechanism for regulating the hypertrophic response. Conversely, deletion of Hdac3 in cardiomyocytes resulted in robust cardiac hypertrophy from metabolic derangements. Furthermore, cardiac-specific overexpression of Hdac2 induces significant hypertrophy by 8 weeks of age (18), whereas $\alpha$-MHC-Hdac3 transgenic mice showed no basal phenotype, but did exhibit stressdependent cardiomegaly (data not shown). Additionally, we generated cardiac-specific transgenics for $\mathrm{Hdac} 1$ and $\mathrm{Hdac} 2$, and these mice showed robust cardiac hypertrophy, cardiac dilatation, and sudden death, dependent upon the level of overexpression (data not shown). Together, these studies illustrate the contrasting roles for $\mathrm{Hdac} 1 / \mathrm{Hdac} 2$ and $\mathrm{Hdac} 3$ in cardiomyopathies and show that class I HDACs serve as distinct nodal points in the precise regulation of gene expression to maintain cardiac function.

Implications for human disease. Class I HDAC inhibitors have shown benefits in a variety of disease states, and the first HDAC inhibitor has just been granted approval by the FDA for cutaneous $T$ cell lymphoma (45). Recent studies have implicated class I HDACs as the primary target of broad-spectrum HDAC inhibitors in vivo; however, the specificity of HDAC inhibitors for class I HDACs has remained enigmatic. Current class I HDAC inhibitors do not show significant specificity for HDAC1 and HDAC2 over HDAC3. Given the widespread efforts to develop HDAC inhibitors for numerous disorders, the phenotype of Hdac 3 mutant mice emphasizes the need to avoid HDAC3 inhibition so as to avoid cardiac toxicity.

\section{Methods}

Generation of a conditional Hdac3 allele. An Hdac3-targeting vector was generated using the pGKNEO-F2L2-DTA vector. This vector contains a neomycin resistance cassette flanked by frt and loxP sites and a diphtheria toxin gene cassette. The arms for homologous recombination were generated by high-fidelity PCR amplification (Expand High Fidelity PCR System; Roche) of 129SvEv genomic DNA. The targeting vector was linearized with PvuI and electroporated into 129SvEv-derived ES cells. 500 ES cell clones were screened for homologous recombination by Southern blot analysis. Genomic DNA was digested with BamHI, and successful loxP site incorporation was confirmed with both a $5^{\prime}$ and a $3^{\prime}$ probe. Targeted ES cells were injected into the blastocysts of C57BL/ 6 females to generate chimeric mice. Chimeras were bred to $\mathrm{C} 57 \mathrm{BL} / 6$ females to achieve germ-line transmission.

Histology, immunohistochemistry, and electron microscopy. Tissues were fixed in $4 \%$ paraformaldehyde, embedded in paraffin, and sectioned at $5-\mu \mathrm{m}$ intervals.
Sections were stained with $\mathrm{H} \& \mathrm{E}$ or Masson trichrome using standard procedures. For neutral lipid staining, hearts were fixed in $4 \%$ paraformaldehyde, cryoembedded, stained with oil red $\mathrm{O}$, and counterstained with hematoxylin. For transmission electron microscopy, LV tissue was minced and fixed in $2 \%$ paraformaldehyde, $2.5 \%$ glutaraldehyde, and $0.1 \mathrm{M}$ cacodylate buffer, prepared according to standard protocol; and electron microscopy was performed at the University of Texas Southwestern Molecular and Cellular Imaging Facility using a Tecnai G2 Spirit 120 KV TEM.

RT-PCR and microarray. For all gene expression analyses from wild-type and Hdac3cko mice, 4 wild-type and 4 Hdac3cko hearts were isolated and analyzed. Total RNA was purified using TRIzOL reagent according to manufacturer's instructions. For RT-PCR, total RNA was used for reverse transcriptase using random hexamer primers. For primer sequences, see Supplemental Data. Quantitative real-time PCR was performed using TaqMan probes purchased from ABI. For microarray, RNA was extracted from either 3 wild-type or Hdac3cko hearts and subsequently pooled prior to analysis. Microarray analysis was performed using the Mouse Genome 430 2.0 Array (Affymetrix). All heart RNA was from ventricle tissue only. cDNA from multiple settings of cardiac pathology was provided by Eva van Rooij and prepared according to standard protocols (46, 47). Microarray analysis was performed by the University of Texas Southwestern Microarray Core Facility using the Mouse Genome 430 2.0 Array (Affymetrix) as described (48).

ChIP. Neonatal rat myocytes were prepared as described (13). After 24 hours induction, chromatin was harvested as described (49). In brief, cells were formaldehyde cross-linked and lysed, and chromatin was sheared by sonication to approximately 500-bp fragments. Sheared chromatin was immunoprecipitated with antibodies against HA (Sigma-Aldrich), HDAC3 (Abcam), or PPAR $\alpha$ (Santa Cruz Biotechnology Inc.), and DNA was isolated and analyzed by PCR with primers flanking binding sites for the indicated response element. For primer sequences, see Supplemental Data. For quantitative ChIP, myocytes were isolated from wild-type and Hdac3cko hearts and chromatin was isolated as described. Sheared chromatin was immunoprecipitated with Acetyl-H3 (Upstate), and DNA was analyzed as before. Error bars represent each reaction performed in triplicate. Similar results were obtained when the experiment was repeated.

Echocardiography. Cardiac function was analyzed by $2 \mathrm{D}$ echocardiography on nonsedated mice using a VingMed System (GE VingMed Ultrasound) and a $11.5-\mathrm{MHz}$ linear array transducer. M-mode tracings were used to measure anterior and posterior wall thicknesses at end diastole and end systole. LVID was measured as the largest anteroposterior diameter (LVIDd or LVIDs). A single observer blinded to mouse genotypes analyzed the data. LV fractional shortening (FS) was calculated according to the following formula: FS $(\%)=[($ LVIDd - LVIDs $) /$ LVIDd $] \times 100$.

Electrocardiography. ECG was performed on sedated adult mice using Accutac Diaphoretic ECG Electrodes (ConMed Corp.). Pads were attached to all 4 limbs, and leads I, II, III, aVR, aVL, and aVF were recorded using PageWriter XLs (Hewlett Packard). Traces were recorded using identical settings for wild-type and Hdac3cko mice $(50 \mathrm{~mm} / \mathrm{s} ; 20 \mathrm{~mm} / \mathrm{mV})$.

Western blotting and histone isolation. Heart tissue was homogenized in lysis buffer $(50 \mathrm{mM}$ Tris at $\mathrm{pH} 7.4,150 \mathrm{mM} \mathrm{NaCl}, 1 \%$ Triton X-100, $1 \mathrm{mM}$ EDTA) supplemented with protease inhibitors (Complete Mini, EDTAfree; Roche) and centrifuged at $14,000 \mathrm{~g}$ for 5 minutes, and supernatant was recovered. $10 \mu \mathrm{g}$ of protein was resolved by SDS-PAGE on a $10 \%$ acrylamide gel and analyzed by Western blot using antibodies against HDAC3 (rabbit polyclonal, 1:1000; Abcam) or eIF5 (rabbit polyclonal, 1:1000; Santa Cruz Biotechnology Inc.) as a loading control.

Histones were extracted from heart tissues using standard procedures. In brief, heart tissue was disrupted with a pestle in PBS containing $0.5 \%$ Triton $\mathrm{X}-100,1 \mathrm{mM}$ PMSF, and $3 \mu \mathrm{M}$ trichostatin A. Nuclei were pelleted, resus- 
pended in $0.4 \mathrm{~N} \mathrm{H}_{2} \mathrm{SO}_{4}$, and extracted for 2 hours at $4^{\circ} \mathrm{C}$. Histones were precipitated by adding $\times 10$ ice-cold acetone and incubating at $-20^{\circ} \mathrm{C}$ for 2 hours; after centrifugation, they were resuspended in water by sonification. Western blot on isolated histones was performed with the following antibodies: acetyl-H3 (Upstate), acetyl-H4 (Upstate), poly-acetyl-lysine (Cell Signaling Technology Inc.), and histone H3 (Cell Signaling Technology Inc.).

Myocardial triglyceride levels. Lipids were extracted from ventricular tissue using a modified Bligh and Dyer technique. In brief, tissue was homogenized in an ice-cold chloroform/methanol/water (2:1:0.8) solution. Additional chloroform and water was added to separate layers, and the mixture was centrifuged at $12,000 \mathrm{~g}$. Following centrifugation, the chloroform layer was extracted and evaporated, and the resultant residue was resuspended in $0.5 \mathrm{ml}$ isopropanol. Triglyceride content was quantified using a triglyceride quantification kit (Sigma-Aldrich).

Animal studies. For fasting studies, male Hdac3cko and wild-type littermates were fasted for 24 hours. After 24 hours, mice were sacrificed, and hearts were extracted and prepared for oil red $\mathrm{O}$ staining. Control mice were allowed standard chow ad libitum. Wy14,643 (BIOMOL International Inc.) $(50 \mathrm{mg} / \mathrm{kg}$ $\mathrm{BW}$ ) in $50 \% \mathrm{DMSO} /$ saline was administered as a single intraperitoneal injection. Control littermates were administered vehicle, and hearts were extracted 8 hours after injection. All animal studies were approved by the IACUC of the University of Texas Southwestern Medical Center.

Mitochondrial function assays. Eight-week-old wild-type and Hdac3cko hearts were perfused in mannitol sucrose buffer and snap-frozen under liquid nitrogen. Mitochondrial function assays were performed as described (50).

Statistics. Values are presented as \pm SEM unless otherwise noted. Gene expression was normalized to $18 \mathrm{~S}$ ribosomal RNA and calculated as rela-

tive change. Statistics were calculated with Excel (Microsoft). $P<0.05$ was considered statistically significant.

Note added in proof. After completion of this study, a report of the role of $\mathrm{Hdac} 3$ in the control of metabolic gene expression in liver appeared (51). The findings of that study are consistent with those of the present study.

\section{Acknowledgments}

We thank Jose Cabrera for graphics, Jennifer Brown for editorial assistance, and Cheryl Nolen, John Shelton, and Xiang Luo for technical assistance. We are also thankful to Joseph Hill and Herman May for telemetry assays, Laurie Mueller for assistance with electron microscopy, and Eva van Rooij for cardiac pathology samples. We are also grateful to Nik Munshi and Hesham Sadek for helpful discussions. This work was supported by grants from the NIH, the D.W. Reynolds Clinical Cardiovascular Research Center, and the Robert A. Welch Foundation. M. Haberland was supported by a grant from the Deutsche Forschungsgemeinschaft (DFG, HA 3335/2-1).

Received for publication April 4, 2008, and accepted in revised form August 19, 2008.

Address correspondence to: Eric N. Olson, Department of Molecular Biology, University of Texas Southwestern Medical Center, 5323 Harry Hines Boulevard, Dallas, Texas 75390-9148, USA. Phone: (214) 648-1187; Fax: (214) 648-1196; E-mail: eric.olson@ utsouthwestern.edu.

1. Gardin, J.M., and Lauer, M.S. 2004. Left ventricular hypertrophy: the next treatable, silent killer? JAMA. 292:2396-2398.

2. Devereux, R.B., et al. 2004. Prognostic significance of left ventricular mass change during treatment of hypertension. JAMA. 292:2350-2356.

3. Okin, P.M., et al. 2004. Regression of electrocardiographic left ventricular hypertrophy during antihypertensive treatment and the prediction of major cardiovascular events. JAMA. 292:2343-2349.

4. Hill, J.A., and Olson, E.N. 2008. Cardiac plasticity. N. Engl. J. Med. 358:1370-1380.

5. Olson, E.N., and Schneider, M.D. 2003. Sizing up the heart: development redux in disease. Genes Dev. 17:1937-1956.

6. Frey, N., and Olson, E.N. 2003. Cardiac hypertrophy: the good, the bad, and the ugly. Annu. Rev. Physiol. 65:45-79.

7. McKinsey, T.A., and Olson, E.N. 2005. Toward transcriptional therapies for the failing heart: chemical screens to modulate genes. J. Clin. Invest. 115:538-546.

8. Jenuwein, T., and Allis, C.D. 2001. Translating the histone code. Science. 293:1074-1080.

9. Roth, S.Y., Denu, J.M., and Allis, C.D. 2001. Histone acetyltransferases. Annu. Rev. Biochem. 70:81-120.

10. McKinsey, T.A., and Olson, E.N. 2004. Cardiac histone acetylation - therapeutic opportunities abound. Trends Genet. 20:206-213.

11. McKinsey, T.A., Zhang, C.L., Lu, J., and Olson, E.N. 2000. Signal-dependent nuclear export of a histone deacetylase regulates muscle differentiation. Nature. 408:106-111.

12. Zhang, C.L., et al. 2002. Class II histone deacetylases act as signal-responsive repressors of cardiac hypertrophy. Cell. 110:479-488.

13. Antos, C.L., et al. 2003. Dose-dependent blockade to cardiomyocyte hypertrophy by histone deacetylase inhibitors. J. Biol. Chem. 278:28930-28937.

14. Kee, H.J., et al. 2006. Inhibition of histone deacetylation blocks cardiac hypertrophy induced by angiotensin II infusion and aortic banding. Circu- lation. 113:51-59.

15. Kong, Y., et al. 2006. Suppression of class I and II histone deacetylases blunts pressure-overload cardiac hypertrophy. Circulation. 113:2579-2588.

16. Grozinger, C.M., and Schreiber, S.L. 2002. Deacetylase enzymes: biological functions and the use of small-molecule inhibitors. Chem. Biol. 9:3-16.

17. Montgomery, R.L., et al. 2007. Histone deacetylases 1 and 2 redundantly regulate cardiac morphogenesis, growth, and contractility. Genes Dev. 21:1790-1802.

18. Trivedi, C.M., et al. 2007. Hdac2 regulates the cardiac hypertrophic response by modulating Gsk3 beta activity. Nat. Med. 13:324-331.

19. Guenther, M.G., Barak, O., and Lazar, M.A. 2001. The SMRT and $\mathrm{N}$-CoR corepressors are activating cofactors for histone deacetylase 3. Mol. Cell. Biol. 21:6091-6101.

20. Sakai, K., and Miyazaki, J. 1997. A transgenic mouse line that retains Cre recombinase activity in mature oocytes irrespective of the cre transgene transmission. Biochem. Biophys. Res. Commun. 237:318-324.

21. Agah, R., et al. 1997. Gene recombination in postmitotic cells. Targeted expression of Cre recombinase provokes cardiac-restricted, site-specific rearrangement in adult ventricular muscle in vivo. J. Clin. Invest. 100:169-179.

22. Gui, C.Y., Ngo, L., Xu, W.S., Richon, V.M., and Marks, P.A. 2004. Histone deacetylase (HDAC) inhibitor activation of $\mathrm{p} 21 \mathrm{WAF} 1$ involves changes in promoter-associated proteins, including HDAC1. Proc. Natl. Acad. Sci. U. S. A. 101:1241-1246.

23. Huss, J.M., and Kelly, D.P. 2004. Nuclear receptor signaling and cardiac energetics. Circ. Res. 95:568-578.

24. Finck, B.N., et al. 2002. The cardiac phenotype induced by PPAR $\alpha$ overexpression mimics that caused by diabetes mellitus. J. Clin. Invest. 109:121-130.

25. Young, M.E., et al. 2001. Uncoupling protein 3 transcription is regulated by peroxisome proliferator-activated receptor (alpha) in the adult rodent heart. FASEB J. 15:833-845.

26. Stanley, W.C., Lopaschuk, G.D., and McCormack, J.G. 1997. Regulation of energy substrate metabo- lism in the diabetic heart. Cardiovasc. Res. 34:25-33.

27. Glyn-Jones, S., et al. 2007. Transcriptomic analysis of the cardiac left ventricle in a rodent model of diabetic cardiomyopathy: molecular snapshot of a severe myocardial disease. Physiol. Genomics. 28:284-293.

28. Murthy, V.K., and Shipp, J.C. 1977. Accumulation of myocardial triglycerides ketotic diabetes; evidence for increased biosynthesis. Diabetes. 26:222-229.

29. Boudina, S., and Abel, E.D. 2007. Diabetic cardiomyopathy revisited. Circulation. 115:3213-3223.

30. Boudina, S., and Abel, E.D. 2006. Mitochondrial uncoupling: a key contributor to reduced cardiac efficiency in diabetes. Physiology (Bethesda). 21:250-258.

31. Cocco, T., Di Paola, M., Papa, S., and Lorusso, M. 1999. Arachidonic acid interaction with the mitochondrial electron transport chain promotes reactive oxygen species generation. Free Radic. Biol. Med. 27:51-59.

32. Loskovich, M.V., Grivennikova, V.G., Cecchini, G., and Vinogradov, A.D. 2005. Inhibitory effect of palmitate on the mitochondrial NADH:ubiquinone oxidoreductase (complex I) as related to the active-de-active enzyme transition. Biochem. J. 387:677-683

33. Schonfeld, P., and Reiser, G. 2006. Rotenone-like action of the branched-chain phytanic acid induces oxidative stress in mitochondria. J. Biol. Chem. 281:7136-7142.

34. Guan, H.P., Ishizuka, T., Chui, P.C., Lehrke, M., and Lazar, M.A. 2005. Corepressors selectively control the transcriptional activity of PPARgamma in adipocytes. Genes Dev. 19:453-461.

35. Fajas, L., et al. 2002. The retinoblastoma-histone deacetylase 3 complex inhibits PPARgamma and adipocyte differentiation. Dev. Cell. 3:903-910.

36. Kersten, S., et al. 1999. Peroxisome proliferatoractivated receptor alpha mediates the adaptive response to fasting. J. Clin. Invest. 103:1489-1498.

37. Watanabe, K., et al. 2000. Constitutive regulation of cardiac fatty acid metabolism through peroxi- 
some proliferator-activated receptor alpha associated with age-dependent cardiac toxicity. J. Biol. Chem. 275:22293-22299.

38. Cheng, L., et al. 2004. Cardiomyocyte-restricted peroxisome proliferator-activated receptor-delta deletion perturbs myocardial fatty acid oxidation and leads to cardiomyopathy. Nat. Med. 10:1245-1250.

39. Burkart, E.M., et al. 2007. Nuclear receptors PPARbeta/delta and PPARalpha direct distinct metabolic regulatory programs in the mouse heart. J. Clin. Invest. 117:3930-3939.

40. Huss, J.M., Torra, I.P., Staels, B., Giguere, V., and Kelly, D.P. 2004. Estrogen-related receptor alpha directs peroxisome proliferator-activated receptor alpha signaling in the transcriptional control of energy metabolism in cardiac and skeletal muscle. Mol. Cell. Biol. 24:9079-9091.

41. Villagra, A., et al. 2007. Histone deacetylase 3 downregulates cholesterol synthesis through repression of lanosterol synthase gene expression. J. Biol. Chem. 282:35457-35470.

42. Fischle, W., et al. 2002. Enzymatic activity associated with class II HDACs is dependent on a multiprotein complex containing HDAC3 and SMRT/ N-CoR. Mol. Cell. 9:45-57.

43. Gregoire, S., et al. 2007. Histone deacetylase 3 interacts with and deacetylates myocyte enhancer factor 2. Mol. Cell. Biol. 27:1280-1295.

44. Kim, Y., et al. 2008. The MEF2D transcription factor mediates stress-dependent cardiac remodeling in mice. J. Clin. Invest. 118:124-132.

45. Duvic, M., et al. 2007. Phase 2 trial of oral vorinostat (suberoylanilide hydroxamic acid, SAHA) for refractory cutaneous T-cell lymphoma (CTCL). Blood. 109:31-39.

46. van Rooij, E., et al. 2008. Dysregulation of microRNAs after myocardial infarction reveals a role of miR-29 in cardiac fibrosis. Proc. Natl. Acad. Sci. U. S. A.
105:13027-13032.

47. van Rooij, E., et al. 2006. A signature pattern of stress-responsive microRNAs that can evoke cardiac hypertrophy and heart failure. Proc. Natl. Acad. Sci. U. S. A. 103:18255-18260.

48. Davis, C.A., et al. 2006. PRISM/PRDM6, a transcriptional repressor that promotes the proliferative gene program in smooth muscle cells. Mol. Cell. Biol. 26:2626-2636.

49. Nelson, J.D., Denisenko, O., Sova, P., and Bomsztyk, K. 2006. Fast chromatin immunoprecipitation assay. Nucleic Acids Res. 34:e2.

50. Yu, X., et al. 2007. Early myocardial dysfunction in streptozotocin-induced diabetic mice: a study using in vivo magnetic resonance imaging (MRI). Cardiovasc. Diabetol. 6:6

51. Knutson, S.K., et al. 2008. Liver-specific deletion of histone deacetylase 3 disrupts metabolic transcriptional networks. EMBO J. 27:1017-1028. 